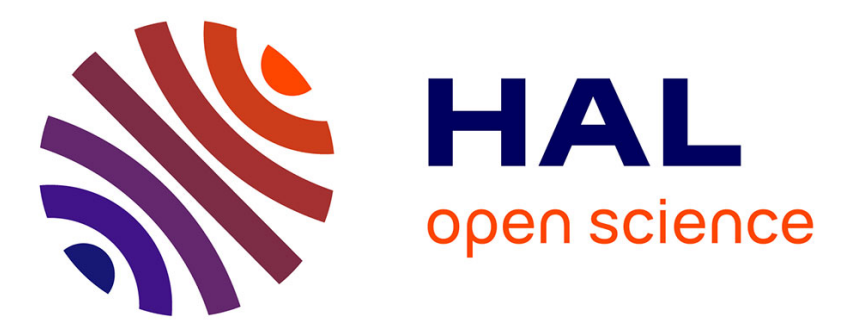

\title{
Incipient Sliding Analysis Between Two Contacting Bodies. Critical Analysis of Friction Law
}

Thami Zeghloul, Marie-Christine Baietto, Bernard Villechaise

\section{To cite this version:}

Thami Zeghloul, Marie-Christine Baietto, Bernard Villechaise. Incipient Sliding Analysis Between Two Contacting Bodies. Critical Analysis of Friction Law. Science direct, 1994, 27 (C), pp.549-557. 10.1016/S0167-8922(08)70340-7 . hal-01951922

\section{HAL Id: hal-01951922 \\ https://hal.science/hal-01951922}

Submitted on 6 Jul 2021

HAL is a multi-disciplinary open access archive for the deposit and dissemination of scientific research documents, whether they are published or not. The documents may come from teaching and research institutions in France or abroad, or from public or private research centers.
L'archive ouverte pluridisciplinaire HAL, est destinée au dépôt et à la diffusion de documents scientifiques de niveau recherche, publiés ou non, émanant des établissements d'enseignement et de recherche français ou étrangers, des laboratoires publics ou privés.

\section{(c)(1)}

Distributed under a Creative Commons Attribution| 4.0 International License 


\section{INCIPIENT SLIDING ANALYSIS BETWEEN TWO CONTACTING BODIES. CRITICAL ANALYSIS OF FRICTION LAW.}

\section{T. ZEGHLOUL*, M.C. DUBOURG* ${ }^{\star}$, B. VILLECHAISE*}

* Université de Poitiers, Laboratoire de Mécanique des Solides, URA CNRS 861, Av. Du Recteur Pineau, 86022 Poitiers Cédex, France.

** INSA, Laboratoire de Mécanique des Contacts CNRS URA 856, Bât. 113, 20 Av. A. Einstein, 69621

Villeurbanne Cédex, France.

Two non-conforming bodies are brought into contact. They touch over a plane surface of extent size compared with the dimensions of the solids. Then they are subjected to a tangential force until gross sliding is observed at the contact interface. Under certain circumstances, this phenomemom is preceeded by local slidings over part of the contact area, named as "sliding waves". They are similar to the Schallamach waves. Relations between these instabilities and the stick limit and the friction conditions on one hand and with the body compliance on the other hand must be investigated as they generate vibratory phenomena like for instance judder.

Progri et al. [1] have identified the experimental conditions for reproducing these phenomena. A two-body contact is established between a rectangular polyurethane slab $(\mathrm{L}=80 \mathrm{~mm}, \mathrm{l}=10 \mathrm{~mm}, \mathrm{~h}=40 \mathrm{~mm}$, Young modulus $\mathrm{E}=7 \mathrm{MPa}$, Poisson's ratio $v=0.48$ ) and an araldite flat, considered as rigid comparatively. Phenomenological description of these phenomena was performed. Mouwakeh et al [2] performed a quantitative analysis and show that the energy dissipated during sliding was comparable with the energy associated with the propagation of a shearing mode interface crack. Numerical simulations based on variational formulations were proposed by numerous authors, and more particularly by Raous [3]. The friction law used is the Coulomb's law. Sliding waves are observed experimentally at one edge of the contact area, sweep through it first partially, then entirely and lead finally to gross sliding. Correct modelling of all stages of the sliding evolution from rest up to gross sliding is still not performed: a central slip zone is predicted, based on the assumption of a friction coefficient equal to the ratio of the tangential load corresponding to the gross sliding over the normal load.

This study deals with these differences. Thus:

- modelling of interface behaviours based on the Coulomb's law were listed. The numerical model is based on a combination of the finite element technique, the interfacial crack theory and the unilateral contact analysis with friction. Geometrical dimensions of the slab and the friction coefficient value have a great influence on the way the sliding stages progress.

- tests were realised to give an accurate phenomenological description of the sliding waves. The influence of both the geometrical dimensions of the slab and the friction coefficient value have been verified. Further both load and displacement variations when the sliding waves sweep through the contact area, partially or totally, were performed. Particularly, informations on the influence of the loading speed on sliding were obtained.

For the configuration studied, incipient slidings are highly dependent on the parameters mentionned above. These results can't be extrapolated directly to industrial configurations due to the difference in material, a very compliant one in tests, rigid ones in industry. But this device is of a great help for local frictional behaviour analysis. Comparisons between experimental and theoretical results and the behaviours noted from the parametric study lead to the conclusion that a parameter adjustement will allow to model sliding stages with a Coulomb law type. 


\section{INTRODUCTION}

A theoretical approach of friction and sliding phenomena between two frictional contacting bodies is presented in this paper. Progri et al [1] and Mouwakeh et al [2] have previously studied experimentally these phenomena and identified conditions leading to "sliding waves" resembling "Schallamach waves" [4] between two contacting bodies. A two-body contact is formed between a rectangular polyurethane slab and an araldite rigid flat (cf. figure 1). The slab is first pressed against the flat, then a tangential load is imposed until gross sliding is detected. Sliding is due to successive pertubations or waves located at the interface and wich travel through it. These pertubations are associated to the propagation of a shearing mode interface crack, as the energy dissipated during sliding and the energy dissipated during the propagation of an interface crack are comparable [2].

Numerical simulations of these experiment based on variational formulations were proposed by Raous [3] (finite element method) and Deshoullieres [5] (boundary integral equations). These simulations are based on the Coulomb's law with static and dynamic friction coefficients: a static coefficient of friction fs defines the "stick limit", ( $\left|\sigma_{\mathrm{nt}}\right|<\mathrm{fs}$ * $\left|\sigma_{\mathrm{nn}}\right|$ ), a dynamic coefficient of friction fd equal to the ratio of the limiting value of the tangential load leading to gross sliding over the normal load- defines the shear stress $\left(\left|\sigma_{\mathrm{nt}}\right|=\mathrm{fd}\right.$ * $\left|\sigma_{\mathrm{nn}}\right|$ ). The stick and slip zone evolutions in the contact are modelled. But the evolution of the state of the contact is still different numerically from the one observed experimentally $[1,2]$. Further a stress field singularity associated to the sliding zone extension is numerically obtained, but less marked than in the experimental case. The Coulomb's friction law and the experimental observations of the contact phenomena disagree. This study aims at :

- listing the different behaviours described by the Coulomb's law. The influence of the coefficient of friction and the slab dimensions will be studied with a parametric study. Location of initial slidings and the direction of extension of the sliding zones will be particularly observed.

- an accurate experimental observation of "the sliding wave" phenomenom.

This model combines numerical and analytical methods. The stress and displacement ficlds are calculated and the stick, slip and open zones at the contact interface are determined. Further the different behaviours at the contact interface depending on the Coulomb's law are identified and listed. This theoretical study is undertaken jointly with an experimental one. A loading frame was developed which allows us to perform accurate

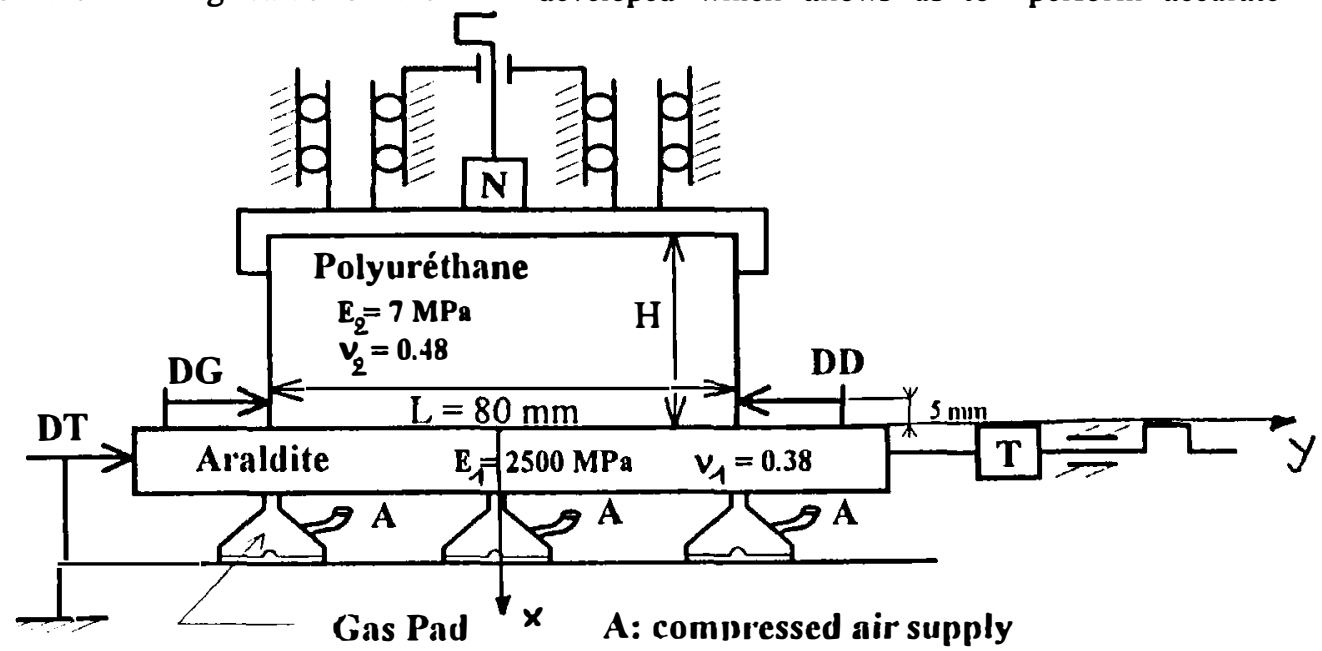

Figure 1 : Experimental device. Mechanical Properties and geometrical dimensions of the contacting bodies 
analysis of the contact evolution during a load cycle. Both loads and displacements are measured continuously and their variations are studied in relation with sliding wave travelling.

\section{MODELLING}

The simplicity of the geometrical shapes of the solids, of their law of behaviour, of the cinematic conditions characterise the experimental configuration. Therefore the following simplifying assumptions are retained for the theoretical modelling:

- steady-state sliding as the sliding speeds are small,

- elastic linearity : the deformable material of the slab behaves homogeneously and isotropically according to Hooke's law,

- two-dimensional and particularly plane strain: the specimens are assumed to be of infinite width compared with the contact dimensions,

- displacements are allowed accross the boundary of the two specimens.

The two-body sliding contact behaviour is particularly difficult to model. A gross sliding event is often preceeded by partial slips over part of the contact surface, causing:

- tangential displacement discontinuities within slip zones,

- normal and tangential displacement discontinuities within open zones,

- continuous normal and tangential displacements within stick zones.

The slip and open zone distribution (number and location) is unknown at priori and changes during loading. This model uses the previous works of Comninou [6] and Dubourg [7]. It is based on the method of continuous distributions of dislocations for modelling the displacement discontinuities at the contact interface and the contact solution for determining the slip and open zone distribution as a unilateral contact problem with friction. The resultant stress and displacement fields are obtained by superposing the continuous and the crack responses to the load. The continuous field $\left(\sigma^{C}\right.$ ) corresponds to sticking at the contact interface, i.e continuity of the normal and tangential displacements, and respects the interface equilibrium. The finite element method is used to calculate this field. The crack field $\left(\sigma^{F}\right)$, corresponding to displacement discontinuities like slip and opening at the contact interface is a corrector field, added when the boundary conditions are no more respected. The resultant field $(\sigma)$ satisfies the boundary conditions listed underneath (7-10). Analytical expressions for the crack field exist in the literature [8]. The coordinate system $(0, x, y)$ is introduced. $x$ is the inner normal at the body 1 contacting interface, $y$ is the tangential component (cf. fig. 1).

$$
\begin{aligned}
& \sigma_{n n}^{F}\left(s_{j}\right)=-\frac{\mu_{1}(2-A-B)}{\pi\left(k_{1}+1\right)} \frac{L}{2} \int_{-1}^{1} \frac{b x\left(r_{i}\right)}{s_{j}-r_{i}} d r \\
& \sigma_{n t}^{F}\left(s_{j}\right)=-\frac{\mu_{1}(2-A-B)}{\pi\left(k_{1}+1\right)} \frac{L}{2} \int_{-1}^{1} \frac{b y\left(r_{i}\right)}{s_{j}-r_{i}} d r \\
& u_{1}\left(s_{j}\right)=\frac{k_{1}(1-A)+1-B}{4\left(k_{1}+1\right)} L \int_{-1}^{s_{j}} b x\left(r_{i}\right) d r
\end{aligned}
$$

$u_{2}\left(s_{j}\right)=-\frac{k_{1}(1+A)+1+B}{4\left(k_{1}+1\right)} L \int_{-1}^{s_{j}} b x\left(r_{i}\right) d r$

$v_{1}\left(s_{j}\right)=\frac{k_{1}(1-A)+1-B}{4\left(k_{1}+1\right)} L \int_{-1}^{s_{j}} b y\left(r_{i}\right) d r$

$v_{2}\left(s_{j}\right)=-\frac{k_{1}(1+A)+1+B}{4\left(k_{1}+1\right)} L \int_{-1}^{s_{j}} b y\left(r_{i}\right) d r$

with

$A=\frac{1-\Gamma}{1+\Gamma k_{1}} \quad B=\frac{k_{2}-\Gamma k_{1}}{k_{2}+\Gamma} \quad \Gamma=\frac{\mu_{2}}{\mu_{1}}$

$\mathrm{k}_{\mathrm{i}}=3-4 \mathrm{v}_{\mathrm{i}}$

$k_{i}=\left(3-v_{i}\right) /\left(1+v_{i}\right)$

plane deformation

plane stress

where the indices 1 and 2 correspond respectively to the flat and the slab, $L$ is the contact width, bx and by are the Burgers vectors corresponding to open 
and slip. Integration and observation points $r_{i}$ and $s_{j}$ are given by:

$$
\begin{array}{ll}
r_{i}=\cos \left(\frac{2 i-1}{2 n+1} p\right) & i=1, n \\
s_{j}=\cos \frac{2 j p}{2 n+1} & j=1, n
\end{array}
$$

where $\mathbf{n}$ is the number of discretisation points.

Stress and displacement expressions are determined from Airy stress functions, and satisfy therefore automatically to the compatibility and equilibrium equations. Further the stress field singularity at crack tip is correctly taken into account.

The contact interface state is determined numerically by solving the unilateral contact analysis with friction. The boundary conditions for a model using the Coulomb's friction law are the following :

Normal or opening problem $(\mathrm{N})$

$$
\begin{array}{clc}
\text { Contact zone } & \sigma_{n n} \leq 0 \quad \Delta u=0 \\
\Delta u=u_{1}-u_{2} & \\
\text { Open zone } & \sigma_{n n}=0 \quad \Delta u>0
\end{array}
$$

Tangential problem (T)

$$
\begin{gathered}
\text { Stick zone } \quad\left|\sigma_{\mathrm{nt}}\right|<f s^{*}\left|\sigma_{\mathrm{nn}}\right| \Delta v=0 \\
\Delta \mathbf{v}=\mathbf{v}_{1}-\mathrm{v}_{2}
\end{gathered}
$$

Slip zone $\left|\sigma_{\mathrm{nt}}\right|<f d^{*}\left|\sigma_{\mathrm{nn}}\right| \quad \Delta v^{*} \sigma_{\mathrm{nt}} \geq 0$ (10)

where $\sigma_{\mathrm{nn}}$ is the normal stress, $\sigma_{\mathrm{nt}}$ the tangential stress, $\Delta \mathrm{u}$ the opening, $\Delta \mathrm{v}$ the slip and fs and fd the static and dynamic friction coefficients.

The algorithm for the contact problem solution follows the method developed by kalker [9] for twobody in rolling contact and Dubourg [7] for the interfacial crack contact. The contact problem is split in two parts, the normal or opening problem (N) and the tangential one (T). In the normal problem, the contact and open zone distribution along the interface is determined. The stick and slip zone distribution along the contact zone is then determined by the tangential contact solution. (N) and $(\mathrm{T})$ are solved in turn until convergence is reached, i.e when the distribution of open, stick and slip zones is stabilized from one iteration to the next.

\section{NUMERICAL RESULTS}

The starting point for studying the contact interface behaviour is to know the location of the first sliding, as this event is determinant for the interface behaviour. Location of the first sliding is predicted in previous studies [11] by the Coulomb's law depending on a stick threshold and slab dimensions $\mathrm{H} / \mathrm{L}$. A normal displacement is first imposed. It corresponds to a normal load of about $76 \mathrm{~N}$. Then a tangential displacement from the left to the right is applied. The terms "left" and the "right" correspond to the left and right of the reader. This convention is used in the remainder of the paper. The increment of the tangential load is constant and equal to $2 \mathrm{~N}$ whatever the ratio $\mathrm{H} / \mathrm{L}$ is. The sliding zone evolutions were analysed for numerous contact configurations. The variation range of the parameters considered is :

- friction coefficient: from 0.1 to 1.4 , step 0.01

- ratio $\mathrm{H} / \mathrm{L}$ from 0.1 to 1 , step 0.1

- ratio $\mathrm{H} / \mathrm{L}=1.2$ and 1.5

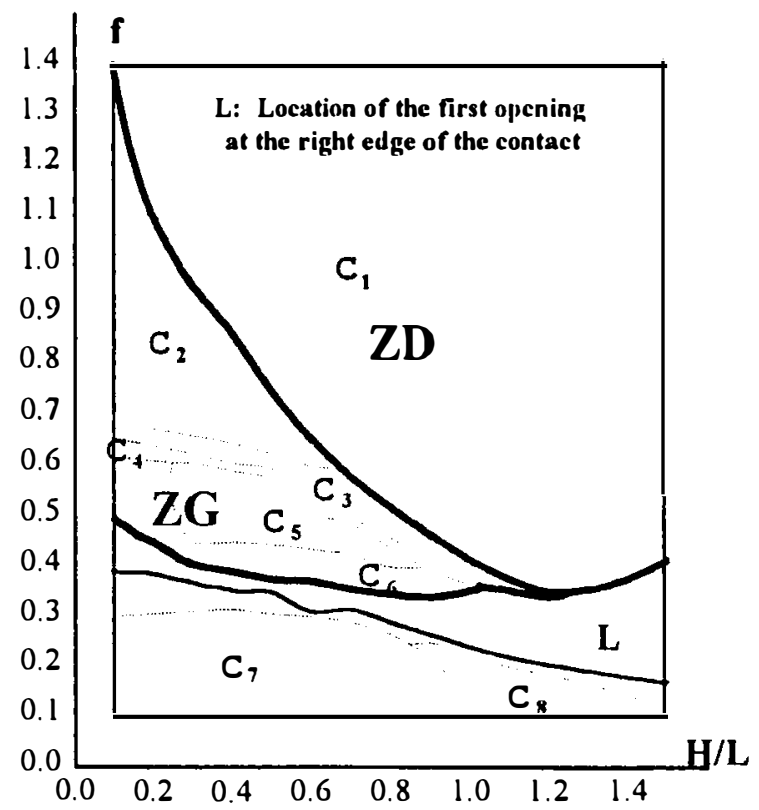

Figure 2 : Contact interface behaviour from numerical simulation based on Coulomb's friction law. 

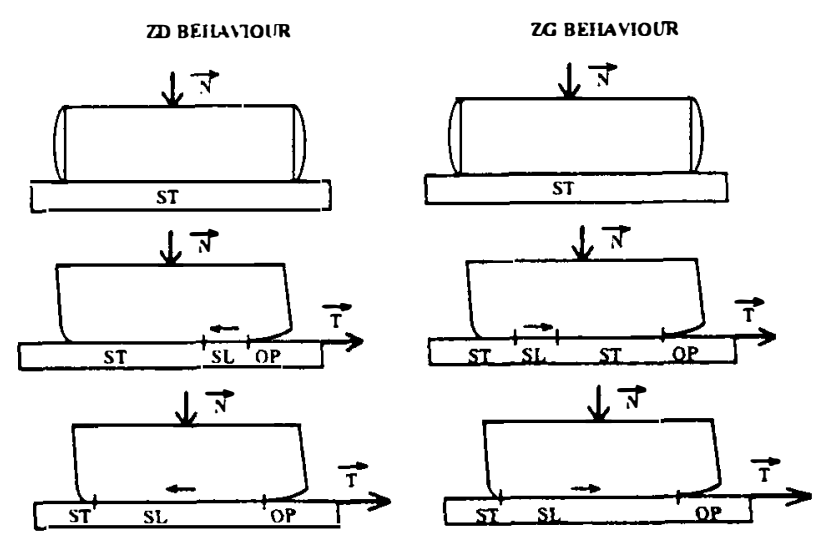

LEGEND

ST : STICK $\quad$ SL: SLIP

OP: OPENDNG

Figure $3:$ ZD and ZG behaviours

Eight zones of behaviour, labelled $\mathrm{Cl}$ to $\mathrm{C} 8$, are obtained from this wide parametric study (1572 cases) (fig. 2).

Each zone corresponds to a typical behaviour of the interface during the loading, i.e identical succession of events (sliding, sticking and opening) takes place at the interface. The $\mathrm{ZD}$ and $\mathrm{ZG}$ behaviour.are presented in detail in Figure 3. The other ones are described in [12]. ZD is related to a sliding zone sweeping through the interface from the right to the left, and ZG is related to a sliding zone starting at the middle of the interface and sweeping through it to the right. This latter point was described in previous numerical studies $[4,5]$.

It comes out from this parametric study that the Coulomb's law covers a wide range of sliding phenomena that depend strongly on parameters non directly linked with the interface. The numerical $Z D$ behaviour seems to correspond to the experimental one on the basis of the analogy between the numerical sliding zones and the experimental sliding waves. It seems therefore possible to reproduce the sliding wave phenomenom by fitting the Coulomb's law.

\section{EXPERIMENTAL PROCEDURE}

An accurate experimental study is neccessary to understand friction and sliding phenomena. Numerical modelling requires a good knowledge of mechanisms, displacement evolution, contact forces and generalized forces. The photoelastic bench includes 4 functional groups, (cf. Figure 4)

- 1: the loading frame, is formed of two parts holding the test specimens. Displacements in two perpendicular directions are feasible. The horizontal guide is performed by two ball columns, the horizontal one by a gas slider. The araldite counterface is mounted on a car differential screw. A sensor force $T$ which controls the tangential force imposes this displacement.

- 2: the optical photoelastic device, allows the continuous observation of the isochromatic field.

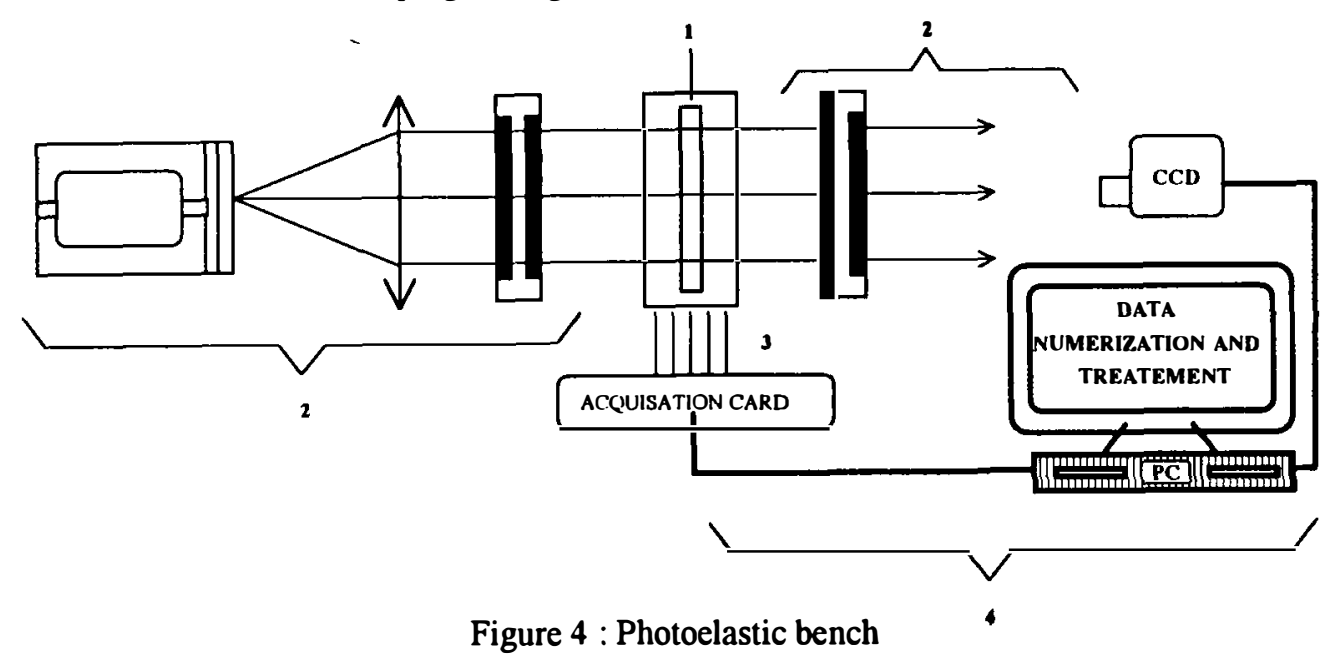



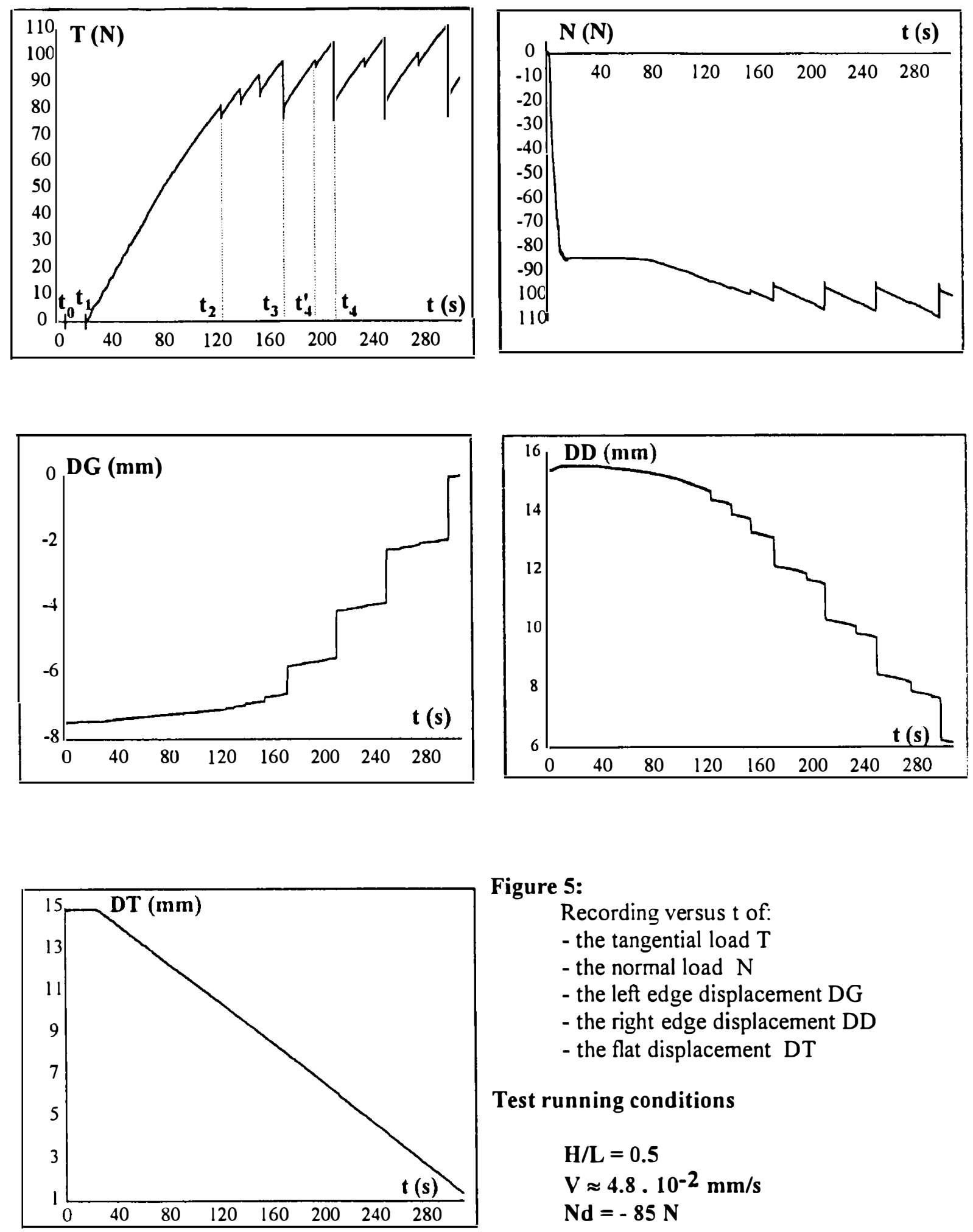

Figure 5:

Recording versus $t$ of:

- the tangential load $\mathrm{T}$

- the normal load $\mathrm{N}$

- the left edge displacement DG

- the right edge displacement DD

- the flat displacement DT

\section{Test running conditions}

$$
\begin{aligned}
& H / L=0.5 \\
& V \approx 4.8 \cdot 10^{-2} \mathrm{~mm} / \mathrm{s} \\
& N d=-85 \mathrm{~N}
\end{aligned}
$$


- 3: load and displacement measurement system, containing

- sensors for normal $(\mathrm{N})$ and tangential $(\mathrm{T})$ load measurements,

- sensors for flat travel (DT) (sliding and slab deflection), for the left edge (DG) and right edge (DD) slab sliding measurements

- 4: measured parameters acquisition and treatement: all signals are recorded and monitored during tests on an informatic system

The picture acquisition for numerical treatement or continuous recording on video tape is performed with the CCD camera.

\subsection{Analysis of a typical test}

Recordings of the normal and tangential loads, the lateral edge displacements DG and DD of the slab and the counterface displacement DT are presented figure 5. Several steps are noted during this test:

- Step 1: loading $t_{0} \leq t \leq t_{1}$. The normal load increases from zero to $\mathrm{Nd}=-85 \mathrm{~N}$. The tangential load is nil and the tangential displacement DT is unchanged. A stick zone holds at the contact interface: there is no difference between the tangential displacements of the slab and the flat. Consequently the shape of the slab is changed into a barrel and the displacement of the lateral edges of the slab DG and DD increase. Note that the displacement sensors are situated $5 \mathrm{~mm}$ above the contact surface. The isochromatic field changes continuously and is symmetric.

- Step 2: complete sticking $t_{1} \leq t \leq t_{2}$. The normal displacement and thus the normal load are kept constant until the end of the test. A tangential displacement is imposed to the right at constant speed V. The tangential load increases progressively and continuously. The interface is still sticking. The flat shift causes defections of the lateral edges of the slab. Theses deflections modify the displacements DD and DG. Continuous evolution of the isochromatic field through the whole slab is observed, due to shearing.
- Step 3: opening - sliding $t_{2} \leq t \leq t_{3}$. An open zone is situated at the right edge of the slab. At $t=t_{2}$, a sliding wave starts at the right side of the slab and sweeps entirely through the contact (cf. figure 6). Tangential load drop is measured as the sliding wave comes out of the interface. The gross sliding is clearly identified by the large variations of the displacements DD and DG. The travel of the sliding wave from one side of the contact to the other is in the opposit direction to those of the imposed tangential displacement. After this event, the interface is again fully adherent. The tangential displacement and therefore the tangential load continue to increase. Three other sweeps are recorded that correspond also to gross slidings. Resulting tangential unloadings and displacement DD and DG variations increase with the number of sweeps. The normal load evolution is similar to those of the tangential load.

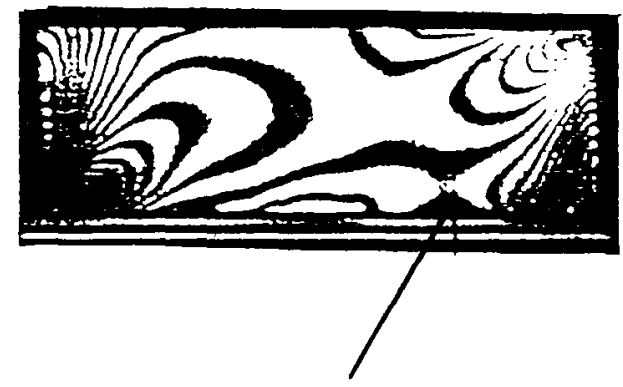

Figure 6 : Sliding wave

- Step 4: partial sliding $t_{3} \leq t \leq t_{4}$ : At $t=t_{4}$, a sliding wave travels partially through the contact interface, but without coming out. A short unloading, weaker than one corresponding to a gross sliding, is recorded. This is confirmed by the displacement DD and DG measurements.

Similar steps are observed up to the end of the test. The maximum tangential load does not vary any more. The tangential load over normal load ratio corresponding to the gross slidings is roughly constant and equal to 1 . 
The interface contact behaviour is summarized in three steps:

- the stick step: during normal loading and for small tangential loads, the interface is completely sticking. There is no energy dissipation and no hysteresis.

- the partial slip and stick step: increasing the tangential load causes sliding waves or Schallamach waves at the contact interface. No gross slidings are observed. This transient step is the connection between the stick step, corresponding to a bulk deformation, and the next step, corresponding to shearing at the interface. Energy is dissipated during this step, which is irreversible.

- the gross sliding step: the sliding waves travel entirely through the contact interface in the opposite direction of the imposed displacement. Gross slidings are observed. A tangential load drop and sticking at the interface are associated to each gross sliding. The stress field in the slab is different before and after this event.

\subsection{Influence of the flat nature}

The sticking conditions and friction at the interface, characterized by a mean friction coefficient, are changed here. Therefore physical and microgeometrical parameters thought influent have been modified. This control is obviously not very accurate as this tribological problem is very complex [10].

Different manufacturings of the surface conditions of the rigid flat were realised. The contact evolution is similar to those described above. The influence of the surface conditions is therefore small on the $T / N$ ratio.

Then different coatings or lubricants were employed to modify the sticking and frictional proporties o the counterface. For instance the flat was coated with paraffin. This third body changes completely the beheviour observed during previous tests. No travel of sliding waves is observed at the contact interface. The speed accomodation was performed within the paraffin layer and not at the interface paraffin/polyurethane.

\section{COMPARISON. CONCLUSION}

The contact evolution during cyclic loading was analysed accurately. The interface behaviour depends on numerous parameters. Their importance and role are sometimes still misunderstood. Two kinds of contact evolution, characterized by the location and the travelling direction of the sliding waves, are highlighted in this study. Numerical results show that the contact interface behaves differently depending on parameters non directly linked to the interface. Nevertheless an analogy is noted between the experimental sliding waves and the theoretical slip zones. Numerical modelling of these phenomena requires important fitting of the parameters that justify modifications of the Coulomb's friction law.

\section{REFERENCES}

1 Progri R., Villechaise B., Godet M. "Fracture mechanics and initial displacements", Mechanisms and surface distress, D. Dowson, C.M. Taylor, M. Godet, D. Berthe Eds., Butterworth London, pp. 47-54, 1986.

2 Mouwakeh M., Villechaise B., Godet M. " Quantitative study of interface sliding phenomena in a two-body contact", Eur. Jnl. Mech., A/Solids, 10, n 5, pp. 545-555, 1991.

3 Raous M., Chabrand P., Lebon F. "Numerical method for frictionnal contact problems and applications", Journ. Mec. théo. et applic., Spécial issue, sup. $\mathrm{n}^{\circ} 1$ to vol. $7,1988, \mathrm{p} .111$ 128.

4 Schallamach A. "How does rubber slide", Wear, 1971, v. 17, p. 301-312.

5 Deshoullieres B. "Contact avec frottement sec entre deux solides. Résolution pour la loi de Coulomb et des lois non-classiques par la méthode des équations intégrales de frontières", Thèse de Doctorat de l'Univ. de Poitiers, 1990, $116 \mathrm{p}$.

6 Comninou M. "The interface crack with friction in the contact zone", Journ. Appl. Mech., 1977, vol. 44, p. 780-781. 
7 Dubourg M. C. "Le contact unilatéral avec frottement le long des fissures de fatigue dans les liaisons mécaniques", Thèse de Doctorat, INSA de Lyon, 1989, $253 \mathrm{p}$.

8 Dundurs J., Mura T. "Interaction between an edge dislocation and a circular inclusion", Joum. Mech. Phys. Solids, 1964, vol. 12, p. 177-189.

9 Kalker J. J. "Two algorithms for the contact problem in elastostatics", Report of the Department of Mathematics and informatics, 1982, Delf, $\mathrm{n}^{\circ} 82-26,8 \mathrm{p}$.

10 Godet $M$. "Aspects mécaniques de la tribologie", 6ème Congrès français de la Mécanique, Lyon, Sept. 5-9, 1983. Paris A.U.M., p. $1.1-1.24$.

11 Zeghloul $T$., Villechaise $B$. "Phénomènes de glissements partiels découlant de l'usage de la loi de Coulomb dans un contact non lubrifié" Matériaux et Techniques, Spécial Tribologie, déc. 1991, p. 10-14.

12 Zeghloul T. "Etude des phénomènes d'adhérences et de glissements dans un contact entre solides: Approche expérimentale et modélisation", Thèse de Doctorat de l'Univ. de Poitiers, 1992, 188 p. 